Rev. Adm. Saúde - Vol. 18, № 72, jul. - set. 2018

http://dx.doi.org/10.23973/ras.72.129

ARTIGO ORIGINAL

\title{
Análise de impacto orçamentário de próteses dispensadas em um hospital do SUS
}

Budgetary impact analysis of artificial limbs dispensed at a SUS hospital

\section{Claudia Barbieri Tait Gandolfi', Carlos Alberto Gabrielli Barreto Campello², Edgard Eduard Engel ${ }^{3}$, Carlos Alberto Grespan Bonacim ${ }^{4}$, Amanda Ribeiro Vieira ${ }^{5}$, Altacílio Aparecido Nunes ${ }^{6}$}

1. Administradora, especialista em gestão hospitalar. Assessora técnica do Hospital das Clínicas da Faculdade de Medicina de Ribeirão Preto da USP (HCFMRPUSP), Ribeirão Preto $\mathrm{SP}$

2. Administrador, doutor em administração. Professor da Faculdade de Economia, Administração e Contabilidade de Ribeirão Preto da USP, Ribeirão Preto SP

3. Médico, doutor em medicina. Responsável pelo Ambulatório de Oncologia Ortopédica e pelo Ambulatório de Amputados do HCFMRPUSP, Ribeirão Preto SP

4. Contador, livre-docente em contabilidade governamental. Professor associado da Faculdade de Economia, Administração e Contabilidade de Ribeirão Preto da USP, Ribeirão Preto SP

5. Administradora. Professora do Instituto Federal de São Paulo, Sertãozinho SP

6. Médico, doutor em medicina tropical. Professor associado do Departamento de Medicina Social da Faculdade de Medicina de Ribeirão Preto da USP, Ribeirão Preto SP

\section{RESUMO}

INTRODUÇÃO: As pessoas com deficiências físicas, frequentemente, necessitam de órteses, próteses e meios auxiliares de locomoção, que são fornecidos, gratuitamente, pelo Sistema Único de Saúde (SUS) em centros credenciados. Porém, as equipes de reabilitação têm dúvidas quanto à real utilização destes equipamentos. OBJETIVO: O objetivo deste trabalho é demonstrar qual é o impacto orçamentário nos recursos públicos da não adesão na utilização das próteses. MÉTODO: Um levantamento demonstrou 
que $37 \%$ dos pacientes não utilizam as próteses recebidas do Hospital das Clinicas da Faculdade de Medicina de Ribeirão Preto da Universidade de São Paulo. Foi realizada uma análise de impacto orçamentário comparando-se o cenário em que é dispensado ao paciente tudo que é prescrito, com um cenário alternativo, em que estes equipamentos seriam entregues somente aos $63 \%$ de pacientes que os utilizam. DISCUSSÃO: Levando-se em conta o considerável valor financeiro aplicado neste programa, recomenda-se o aprofundamento das questões relativas à não adesão ao tratamento com próteses, evitando assim a dispensação para aqueles sabidamente não aderentes e possibilitando que os valores economizados sejam usados em outros programas. CONCLUSÃO: O fornecimento racional de próteses pode gerar melhoria significativa na aplicação dos recursos do SUS. Acredita-se que futuros estudos serão importantes para avaliar fatores individuais preditivos para o uso de próteses e potencialmente otimizar aplicação dos recursos públicos.

Palavras-chave: Avaliação de Tecnologias em Saúde; Avaliação Econômica em Saúde; Prótese de Membro; Reabilitação

\begin{abstract}
INTRODUCTION: Disabled persons frequently need orthotic devices, artificial limbs and artificial means of locomotion, which are provided for free by the Sistema Único de Saúde (SUS) in accredited centres. However, the rehabilitation teams question the real usage of those equipments. OBJECTIVE: This paper aims to demonstrate what is the budgetary impact in public resources when not adhering to the use of artificial limbs. METHOD: A survey shows that $37 \%$ of patients do not use artificial limbs received from Hospital das Clínicas da Faculdade de Medicina de Ribeirão Preto da Universidade de São Paulo. A budgetary analysis has been made, comparing the scenario in which everyting prescribed to the patient is dispensed, with an alternate scenario in which those equipments would be delivered only to $63 \%$ of patients who use them. DISCUSSION: Considering the substantial financial value applied to this program, it is recommended further debate on issues related to non-adherence to the treatment with artificial limbs, thus avoiding a dispensation for those known to be non-adherent and allowing that the saved values may be used in different programs. CONCLUSION: The rational provision of artificial limbs may generate a significant improvement in the application of SUS resources. It is believed that future studies will be important to evaluate predictive individual factors for the use of artificial limbs and potentially optimize the application of public resources.
\end{abstract}

Keywords: Technology Assessment, Biomedical; Health Evaluation; Artificial Limbs; Rehabilitation 


\section{INTRODUÇÃO}

As pessoas com deficiências motoras ressentem-se de uma variedade de condições neurossensoriais que as afetam em termos de mobilidade e de coordenação motora geral. Em muitos casos, conseguem movimentar-se com a ajuda de prótese, cadeira de rodas ou outros aparelhos auxiliares (OPM), considerados, nesta situação, parte integrante do processo de reabilitação.

Os direitos da pessoa com deficiência estão legitimados em leis e decretos federais que buscam cada vez mais a inserção de OPM em políticas públicas de saúde. O gestor estadual de saúde é responsável em promover o acesso gratuito da pessoa com deficiência a estes equipamentos, o que gerou, em 2017, um gasto no valor $R \$ 27.114 .126,25$ no estado de São Paulo com a dispensação de $77.052 \mathrm{OPM}^{(1)}$, valor significativo, porém insuficiente, frente a grande demanda. Estima-se que $30 \%$ da população deste estado tenham algum tipo de deficiência, e que $6,1 \%$, mais de dois milhões e meio de pessoas, apresentem deficiências motoras ${ }^{(2)}$.

Apesar da grande pressão política e judicial para dispensação de OPM, as equipes de reabilitação têm dúvidas quanto à real utilização destes equipamentos. Autores ${ }^{(3)}$ estudaram a adesão ao tratamento de escoliose com órteses equipadas com dispositivo que calculavam o tempo de uso. Concluíram que a adesão foi de apenas $47 \%$ e que as informações dos pacientes e da família em relação ao uso da órtese eram superestimadas ${ }^{(3)}$. Autor ${ }^{(4)}$ pesquisou a adesão ao tratamento com palmilhas para afecções diversas dos pés e concluiu que apenas 15\% apresentaram adesão total e que $47 \%$ abandonaram completamente o seu uso antes do término do tratamento ${ }^{(4)}$.

Após esta análise, imagina-se que é possível que uma parcela significativa dos recursos destinados à compra de OPM não esteja tendo nenhum efeito sobre a reabilitação desta importante parcela da população.

Para verificar a real utilização das OPM entregues pelo Hospital das Clinicas da Faculdade de Medicina de Ribeirão Preto da Universidade de São Paulo (HCFMRPUSP) foi realizado um estudo da adesão ao tratamento ${ }^{(5)}$. Com os resultados obtidos foi realizada uma análise de impacto orçamentário (AIO), que é uma das ferramentas econômicas utilizadas nas avaliações de tecnologia em saúde (ATS). Seu objetivo maior é fornecer, aos responsáveis pela decisão, informações quanto ao potencial impacto e consequências econômicas de determinada tecnologia ou de mudanças na utilização de uma tecnologia $\operatorname{antiga}^{(6)}$.

O crescente conflito entre, por um lado, a necessidade de incorporação de novas tecnologias na atenção à saúde e, por outro lado, a necessidade de adequar o seu uso a recursos financeiros limitados, promoveu a construção e aplicação de instrumentos de apoio à tomada de decisão por gestores de todo o mundo. Países como Canadá, Inglaterra, Austrália e Polônia têm adotado métodos epidemiológicos e econômicos para auxiliar a alocação de recursos financeiros e no planejamento orçamentário. 
Autores $^{(7)}$ informam que no Brasil as iniciativas em ATS começaram a ser inseridas em grupos de pesquisa e instituições acadêmicas mais voltadas para avaliações de programas, seguidas de um discreto movimento pelas avaliações de qualidade e tecnológicas. Nos últimos cinco anos tomou um caráter institucional com ações governamentais, destacando a criação da Rede Brasileira de Avaliação de Tecnologias em Saúde (REBRATS). Atualmente, estudos epidemiológicos e econômicos vêm sendo usados no processo de incorporação de novas tecnologias no Sistema Único de Saúde (SUS), conforme estabelece a lei $n^{\circ} 12.401$ de 28 de abril de $2011^{(7)}$.

\section{OBJETIVOS}

O objetivo deste trabalho é apresentar análise de impacto orçamentário da não adesão na utilização das próteses entregues pelo HCFMRPUSP.

\section{MATERIAL E MÉTODOS}

$\mathrm{Na} A \mathrm{AO}$ é estimado o impacto econômico da incorporação (ou remoção) de uma intervenção considerando-se o conjunto das tecnologias disponíveis para o problema de saúde em análise, incluindo os custos da nova intervenção em si, custos de cointervenções, movimento de recursos associados às opções terapêuticas em uso e possíveis realocações de recursos para os casos que possam resultar em economias ao sistema de saúde ${ }^{(8-11)}$.

A pesquisa realizada com pacientes do HCFMRPUSP ${ }^{(5)}$, demonstrou que $37 \%$ dos pacientes não utilizam as próteses recebidas. Este índice foi utilizado na análise de impacto orçamentário.

Para a realização de uma AIO basta possuir uma referência que, neste caso, seria a manutenção do nível de não utilização apresentado na referida pesquisa, 37\%, comparando-se com a possibilidade de zero de não utilização.

Sendo a prótese considerada uma tecnologia em saúde, enquadra-se perfeitamente no objetivo da $\mathrm{AIO}$, integrando os seguintes elementos:

a) Doença em questão: não é tratada especificamente uma doença e sim uma condição de saúde relacionada à funcionalidade e à incapacidade, identificando o que uma pessoa "pode ou não pode fazer na sua vida diária", tendo em vista as funções dos órgãos ou sistemas e estruturas do corpo, assim como as limitações de atividades e da participação social no meio ambiente onde a pessoa vive. Condições estas que impõem a necessidade de utilização de próteses como facilitadores para o desempenho dessas atividades ${ }^{(12)}$. A Figura 1 demonstra a relação entre esses conceitos.

b) O tratamento em avaliação foi a prótese externa ou não implantada; 
c) A perspectiva analisada foi do SUS através do programa de prescrição e dispensação de próteses do HCFMRPUSP nos anos de 2012, 2013 e 2014 em que foram entregues 351 próteses, sendo 345 de membros inferiores e 6 de membros superiores, cujo investimento foi de $\mathrm{R} \$$ $770.449,83$.

d) A população de referência foram todos os pacientes que receberam próteses neste período através do referido programa.

e) O horizonte temporal proposto foi de 3 anos, considerando sua implantação apenas no HCFMRPUSP.

f) Os cenários comparados foi o que ocorreu de fato, em que houve a dispensação de todas as próteses prescritas no período de 2012 a 2014 e um fictício neste mesmo período, em que foi utilizado o percentual de não utilização.

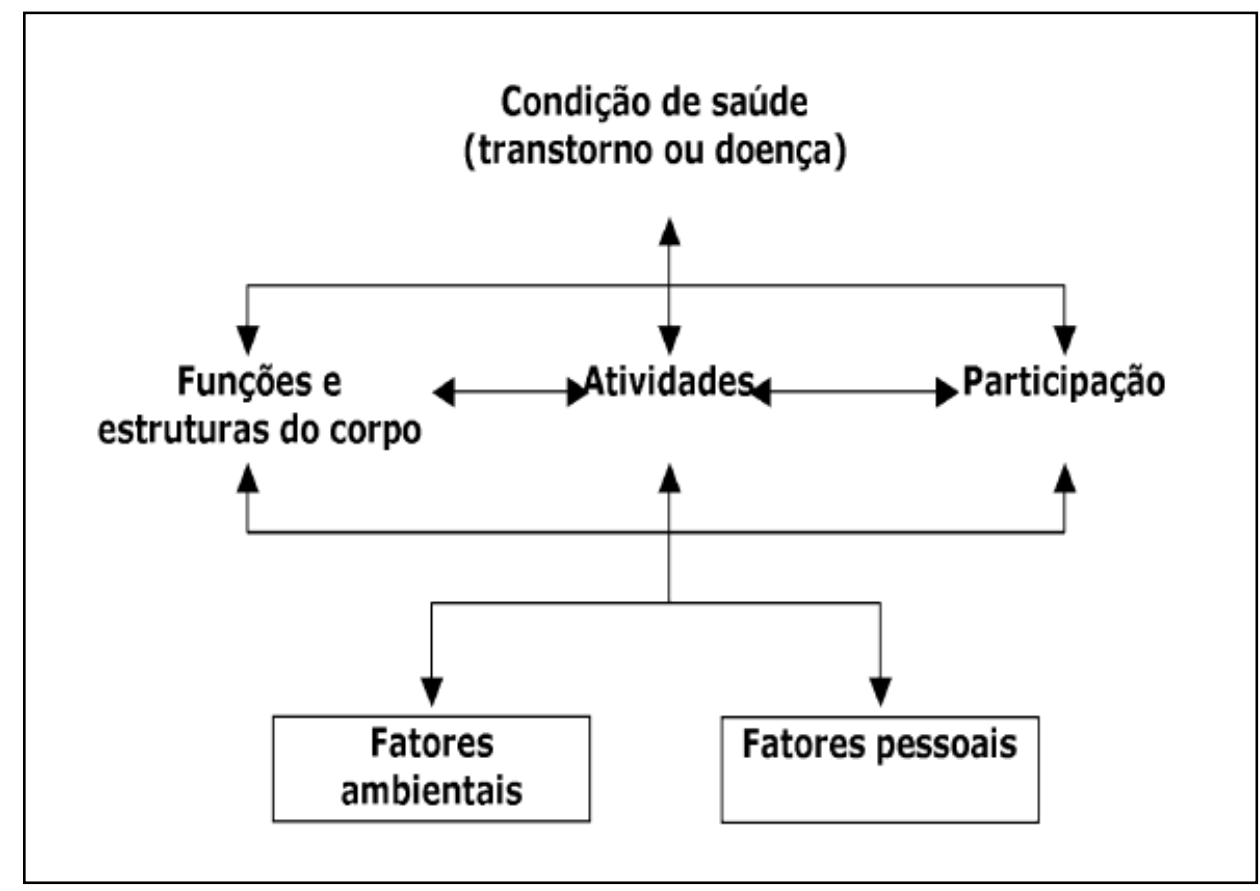

Figura 1. Demonstração gráfica da condição de saúde a ser analisada.

De uma forma simplificada, o custo do tratamento de uma determinada intervenção ou tecnologia consiste em se multiplicar o número de indivíduos doentes com indicação de tratamento pelo custo dos tratamentos que estão sendo avaliados. O impacto orçamentário incremental consiste na diferença de custos entre o cenário do novo tratamento e o cenário de referência (que reflete as práticas terapêuticas atuais, sem a nova intervenção) ${ }^{(13)}$ : 
Impacto orçamentário incremental $=(\mathrm{NiNt} \times \mathrm{CtNt})-(\mathrm{NtA} \times \mathrm{CttA})$

Onde:

$\mathrm{NiNt}=$ Número de indivíduos usando o novo conjunto de tratamentos formado a partir da incorporação da nova intervenção em estudo

$\mathrm{CtNt}=$ Custo total do novo tratamento

$\mathrm{NtA}=$ Número de indivíduos usando o atual conjunto de tratamentos disponível

$\mathrm{CttA}=$ Custo total do tratamento atual

Existem, basicamente, duas formas de se integrar as informações sobre estimativa da população de interesse com a informação das estimativas de custos de forma comparativa entre diferentes cenários: modelos estáticos e modelos dinâmicos.

Neste trabalho foram empregados modelos estáticos, também chamados de determinísticos, que são os mais difundidos e os mais adequados para este estudo, pois o problema de saúde em questão enquadra-se dentro das doenças crônicas e não possui frequentes exacerbações ou remissões.

Para calcular o custo médio de tratamento de um paciente foi observado o Protocolo Assistencial para Atendimento à Pacientes Amputados do Centro de Reabilitação do HCFMRPUSP, em que estão descritos todos os atendimentos que deverão ser realizados durante um tratamento de seis meses, que inclui 0 período pré e pós-protetização.

Os custos do tratamento foram estimados sob duas perspectivas: a primeira considerando o custo médio por procedimento calculado pela Seção de Custos do HCFMRPUSP utilizando o sistema de absorção e a segunda levando em consideração os valores pagos para reabilitação pelo Sistema Único de Saúde obtidos no Sistema de Gerenciamento da Tabela de Procedimentos do SUS $(\text { SIGTAP) })^{(14)}$.

A "finalidade básica da contabilidade de custos é determinar tão exatamente quanto possível, o custo de fabricação de um produto ou prestação de um serviço"(15). Existem diversas formas de custeio que podem ser utilizadas na área da saúde. No HCFMRP USP é utilizado o custeio por absorção, que consiste "no levantamento da totalidade de custos via:

- Identificação de atividades por centro de custo;

- Especificação de recursos consumidos;

- Rateio dos custos indiretos"(16,17) 
Na segunda perspectiva foi considerado o procedimento pago pelo SUS paga o denominado tratamento intensivo de paciente em reabilitação física que consiste no atendimento por equipe multiprofissional e multidisciplinar especializada em reabilitação nas deficiências físicas. o tratamento intensivo compreende um conjunto de atendimentos individuais e/ ou em grupos realizados por equipe multiprofissional e multidisciplinar, realizados ou não no mesmo dia. Inclui, quando necessário, a prescrição, avaliação, adequação, treinamento e acompanhamento da dispensação de órteses, próteses e/ou meios auxiliares de locomoção e orientação familiar ${ }^{(14)}$. É faturado um tratamento intensivo por comparecimento, assim foram considerados 48 atendimentos no período de seis meses, que é o maior número de comparecimentos do paciente.

\section{RESULTADOS}

Conforme já relatado, na pesquisa de adesão foi obtido um resultado geral de não utilização de $37 \%$, percentual utilizado na AIO para a criação de um cenário alternativo.

Nas Tabelas 1, 2 e 3 são apresentados os custos das avaliações e do tratamento, apurados pela Seção de Custos para um período de 6 meses:

Tabela 1. Custos das avaliações apurados pela Seção de Custos para um tratamento de seis meses em reais.

\begin{tabular}{lccc} 
Avaliação & $\begin{array}{c}\text { Número de } \\
\text { Consultas }\end{array}$ & Valor Unitário & Valor Total \\
\hline Fisiatria & 3 & 128,82 & 386,46 \\
Ortopedia & 3 & 128,82 & 386,46 \\
Fisioterapia & 3 & 45,23 & 135,69 \\
Terapia Ocupacional & 3 & 25,24 & 75,72 \\
Serviço Social & 3 & 30,02 & 90,06 \\
Enfermagem & 3 & 32,39 & 97,17 \\
\hline Total & & & $\mathbf{1 . 1 7 1 , 5 6}$ \\
\hline
\end{tabular}

Tabela 2. Custos em reais do tratamento com duração de seis meses apurados pela Seção de Custos considerando que nem todos os pacientes são atendidos por todos os profissionais. 


\begin{tabular}{|c|c|c|c|c|c|c|c|}
\hline Tratamento & $\begin{array}{r}\% \text { de } \\
\text { pacientes } \\
\text { atendidos }\end{array}$ & $\begin{array}{r}\text { № de } \\
\text { comp. } \\
\text { por } \\
\text { semana }\end{array}$ & $\begin{array}{l}\text { № de } \\
\text { sem. }\end{array}$ & $\begin{array}{r}\text { Total } \\
\text { de } \\
\text { atend. }\end{array}$ & $\begin{array}{r}\text { Valor } \\
\text { por } \\
\text { atend. }\end{array}$ & $\begin{array}{r}\text { Valor } \\
\text { total } \\
\text { geral }^{(1)}\end{array}$ & $\begin{array}{r}\text { Valor total } \\
\text { proporcio } \\
\text { nal }\end{array}$ \\
\hline Fisioterapia & $100 \%$ & 2 & 24 & 48 & 26,01 & $1.248,48$ & $1.248,48$ \\
\hline $\begin{array}{l}\text { Terapia } \\
\text { Ocupacional }\end{array}$ & $20 \%$ & 1 & 24 & 24 & 25,24 & 605,76 & 121,15 \\
\hline $\begin{array}{l}\text { Atendimento em } \\
\text { Grupo }\end{array}$ & $100 \%$ & 1 & 24 & 24 & 30,02 & 720,48 & 720,48 \\
\hline Psicólogo & $20 \%$ & 1 & 24 & 24 & 31,02 & 744,48 & 148,90 \\
\hline Serviço Social & $100 \%$ & 2 & 0 & 2 & 30,02 & 60,04 & 60,04 \\
\hline Enfermagem & $50 \%$ & 1 & 24 & 24 & 32,39 & 777,36 & 388,68 \\
\hline Total & & & & & & $4.156,60$ & $2.687,73$ \\
\hline
\end{tabular}

Tabela 3. Resumo dos custos das avaliações e atendimentos apurados pela Seção de Custos para um tratamento de seis meses em reais.

\begin{tabular}{lr} 
Tipo & Valor \\
\hline Avaliações & $1.171,56$ \\
Atendimentos & $2.687,73$ \\
\hline Total & $\mathbf{3 . 8 5 9 , 2 9}$ \\
\hline
\end{tabular}

Na Tabela 4 é apresentado o custo ressarcido pelo MS para um tratamento de seis meses.

Tabela 4. Custo ressarcido pelo MS para um tratamento de seis meses em reais.

\begin{tabular}{lcrr} 
Avaliações & № & Valor Unitário & Valor Total \\
\hline Fisiatria & 3 & 10,00 & 30,00 \\
Ortopedia & 3 & 10,00 & 30,00 \\
Fisioterapia & 3 & 6,00 & 18,00
\end{tabular}


Terapia

3

6,00

18,00

Ocupacional

Serviço Social

3

6,00

18,00

Enfermagem

3

6,00

18,00

Subtotal

132,00

\section{Tratamento}

Fisioterapia

Terapia

Ocupacional

Grupo

Serviço Social

Enfermagem

Tratamento Intensivo
№

48

6

24

2

12

48
Valor Unitário Valor Total

4

.

$21,69 \quad 1.041,12$

De posse destes dados, foi possível a elaboração da análise de impacto orçamentário. Apresentamos abaixo o primeiro cenário, considerando os valores de tratamento apresentados pela Seção de Custos:

Tabela 5. Análise de impacto orçamentário considerando os valores de tratamento apresentados pela Seção de Custos do HCFMRPUSP.

\begin{tabular}{|c|c|c|c|c|}
\hline Cenário $A$ & & & Cenário Alternativo & \\
\hline Próteses & & Valor $(\mathrm{R} \$)$ & Próteses & Valor $(\mathrm{R} \$)$ \\
\hline & 351 & $770.449,83$ & 221 & $485.383,39$ \\
\hline Tratament & de Custos & & Tratamento - Seção & ustos \\
\hline & 351 & $1.354 .610,09$ & 221 & $852.902,65$ \\
\hline CUSTO T & & $\mathrm{R} \$ 2.125 .059,92$ & CUSTO TOTAL & $\mathrm{R} \$ 1.338 .286,04$ \\
\hline
\end{tabular}


$\mathrm{Na}$ Tabela 6 segue o segundo cenário, em que são demonstrados os valores de tratamento ressarcidos pelo MS.

Tabela 6. Análise de impacto orçamentário considerando os valores de tratamento ressarcidos pelo MS disponíveis na Tabela SIGTAP.

\begin{tabular}{|c|c|c|c|}
\hline \multicolumn{2}{|l|}{ Cenário Atual - 100\% } & \multicolumn{2}{|c|}{ Cenário Alternativo - $63 \%$} \\
\hline Próteses & Valor $(\mathrm{R} \$)$ & Próteses & Valor $(\mathrm{R} \$)$ \\
\hline 351 & $770.449,83$ & 221 & $485.383,39$ \\
\hline Tratamento - SIGTAP & & Tratamento - SIGTAP & \\
\hline 351 & $411.765,00$ & 221 & $259.259,52$ \\
\hline CUSTO TOTAL & $\mathrm{R} \$ 1.182 .214,95$ & CUSTO TOTAL & $\mathrm{R} \$ 744.642,91$ \\
\hline
\end{tabular}

\section{DISCUSSÃO}

A AIO sobre a adesão ao tratamento com próteses permitiu aferir o impacto desta prática nas políticas de prescrição e dispensação junto ao SUS. Considerando que $37 \%$ dos pacientes não utilizam as próteses, o resultado é o desperdício de recursos aplicados em cuidados em saúde. Para autores ${ }^{(18)}$ [...] cuidados em saúde representa o somatório de recursos utilizados para a aplicação de uma tecnologia com o objetivo de recuperar ou melhorar o nível de saúde de uma pessoa ou de uma população. Nível de saúde é o estado de saúde em determinado momento do tempo. A falta de entendimento desses dois conceitos, bem como o estabelecimento de correlação direta entre eles, pode gerar decisões equivocadas sobre alocação de recursos" (18).

Autores ${ }^{(19)}$ classificaram as causas de não adesão à diversos tipos de tratamento em quatro grupos: fatores individuais (incluindo variáveis psicológicas e comportamentais), fatores inerentes ao tratamento, fatores interpessoais e fatores ambientais. Mesmo com muitos anos de estudo, pouco se sabe qual é o impacto destes fatores nas atitudes das pessoas. "Para compreender plenamente a adesão, os pesquisadores devem mudar a abordagem para estudar preditores de geral para individual, focando em variáveis que indicam tipos específicos de comportamentos de adesão" (19).

Frente à restrição de recursos, questiona-se a possibilidade de que uma parte de pacientes não atendidos poderia ser beneficiada, caso aqueles que não utilizassem os equipamentos pudessem ser identificados e ao invés de 
receberem, estes fossem dispensados para alguém que se encontra em fila de espera, utilizando para isso os recursos advindos da economia a ser alcançada de $R \$ 285.066,44$ em próteses, somente no HCFMRPUSP.

Além disso, a previsão dos resultados da reabilitação, em particular o uso da prótese, é de grande interesse para os médicos e terapeutas, bem como para as companhias de seguros de saúde, que reembolsam os custos da prótese e do processo de reabilitação, segundo autores ${ }^{(20)}$.

\section{CONCLUSÃO}

Como já mencionado, um dos objetivos da AlO é apontar possíveis realocações de recursos para os casos que possam resultar em economias ao sistema de saúde (08-11). O fornecimento racional de próteses pode gerar melhoria significativa na aplicação dos recursos do SUS.

Futuros estudos serão importantes para avaliar fatores individuais preditivos para o uso de próteses e, potencialmente, otimizar a aplicação dos recursos públicos.

\section{REFERÊNCIAS}

1. DATASUS. Informações de Saúde. Disponível em www.datasus.gov.br. Acesso em: 07 agosto 2018.

2. INSTITUTO BRASILEIRO DE GEOGRAFIA E ESTATÍSTICA. População Residente no Brasil. Disponível em: www.ibge.gov.br. Acesso em: 28 novembro 2012.

3. MORTON, A.; RIDDLE R.; BUCHANAN R.; KATZ D.; BIRCH J. Accuracy in the prediction and estimation of adherence to bracewear before and during treatment of adolescent idiopathic scoliosis. Journal of Pediatric Orthopaedics, v. 28, p. 336-341, 2008.

4. GUIMARÃES, C. Fatores associados à adesão ao uso de palmilhas biomecânicas. Revista Brasileira de Fisioterapia. Rio de Janeiro, v. 10, n. 3, p. 271-277, 2006.

5. ENGEL, E. E. et al. Avaliação da Adesão ao Tratamento com Órteses, Próteses e Meios Auxiliares de Locomoção Dispensados através do Programa de OPM. São Paulo: Processo FAPESP 2012/51224-3, 2012.

6. NEUMANN, P. J. Budget Impact Analyses Get Some Respect. Value in Health, Edinburgh, v. 10, n. 5, p. 324-325, 2007. 
7. POLANCZYK, C. A.; VANNI, T.; KUCHENBECKER R. S. Avaliação de tecnologias em saúde no Brasil e no contexto internacional. In: NITA, M. E. et al. Avaliação de Tecnologias em Saúde: evidência clínica, análise econômica e análise de decisão. São Paulo: Artmed, 2010.

8. MARSHALL D.A.; DOUGLAS P.R.; DRUMMOND M.F.; TORRANCE G.W; MACLEOD S.; MANTI O.; et al. Guidelines for conducting pharmaceutical budget impact analyses for submission to public drug plans in Canada. Pharmacoeconomics, Pennsylvania, v. 26 n. 6, p. 477-495, 2008.

9. UNITED KINGDOM. National Institute for Health and Clinical Excellence. Developing costing tools -Methods guide. London, 2008.

10. AUSTRALIA. Department of Health and Agening. Guidelines for preparing submissions to the Pharmaceutical Benefits Advisory Committee. Camberra, 2006.

11. ORLEWSKA E.; MIERZEJEWSKI P. Proposal of polish guidelines for conducting financial analysis and their comparison to existing guidance on budget impact in other countries. Value Health, Edinburgh, v. 7, p. 1-10, 2004.

12.FARIAS, N.; BUCHALLA C. M. A Classificação Internacional de Funcionalidade, Incapacidade e Saúde da Organização Mundial da Saúde: Conceitos, Usos e Perspectivas. Revista Brasileira de Epidemiologia, São Paulo, v. 8, p. 187-93, 2005.

13.BRASIL. Ministério da Saúde. Diretrizes Metodológicas - Análise de Impacto Orçamentário - Manual para o Sistema de Saúde do Brasil. Brasília, 2012.

14.DATASUS. Sistema de Gerenciamento da Tabela de Procedimentos, Medicamentos e OPM do SUS - SIGTAP. Disponível em www.datasus.gov.br. Acesso em: 28 agosto 2018.

15. TAKATORI, R. S. Desenvolvimento de modelo conceitual de custos padrão e ABC - para o plano de referência básica de uma operadora de plano de assistência à saúde: um estudo de caso. Dissertação, Fundação Escola Álvares Penteado, São Paulo, 2002.

16. NAKAGAWA, M. Gestão estratégica de custos: conceito, sistemas e implementação. São Paulo: Atlas, 1993.

17. MARTIN, K. A.; BOWEN D. J.; DUNBAR-JACOB J.; PERRI M. G. Who will adhere? Key issues in the study and prediction of adherence in randomized controlled trials. Controlled Clinical Trials, New York, v. 21, 195-199, 2000.

18. MEDICI, A.; MARQUES, R. Sistemas de custos como instrumento de eficiência e qualidade dos serviços de saúde. São Paulo: FUNDAP/IESP, 1995.

19. TEICH, N. L.S, Nita, M. E. As medidas de preferências (utilidades) em estados de saúde. São Paulo: Artmed, 2010. 
20.VAN-EIJK, M. S.; VAN DER LINDE, H.; BUIJCK B; GEURTS, A.; ZUIDEMA, S.; KOOPMANS, R. Predicting prosthetic use in elderly patients after major lower limb amputation. Prosthetics and Orthotics International, United Kingdom, v. 36, p. 45-52, 2012.

21.SARTI, F. M.; CYRILLO, D. C. Avaliação de custos em projetos de economia da saúde. In: NITA, M. E. et al. Avaliação de Tecnologias em Saúde: evidência clínica, análise econômica e análise de decisão. São Paulo: Artmed, 2010.

Recebido: 05 de setembro de 2018. Publicado: 28 de setembro de 2018

Correspondência: Claudia Barbieri Tait Gandolfi. E-mail: claudiabtg@hcrp.usp.br

Conflito de Interesses: os autores declararam não haver conflito de interesses.

(C) This is an Open Access article distributed under the terms of the Creative Commons Attribution License, which permits unrestricted use, distribution, and reproduction in any medium, provided the original work is properly cited 